\title{
Surgery of isolated patent ductus arteriosus: a new technique of suture and division
}

\author{
P. NANDI
}

From the University of Hong Kong, Queen Mary Hospital, Hong Kong

Nandi, P. (1977). Thorax, 32, 5-6. Surgery of isolated patent ductus arteriosus: a new technique of suture and division. Since mid-1973 29 cases of isolated patent ductus arteriosus were treated in the Hong Kong University surgical unit with a new technique of suture and division. This technique has almost eliminated the problem of intraoperative haemorrhage and its consequences.

Gross and Hubbard (1939) were the first to report successful ligation of a patent ductus arteriosus. Since then the value of operative treatment of this condition has been universally accepted. Division and suture of the ductus seems to be the method of choice in many centres. When using the standard technique of dividing the ductus between clamps, followed by suturing, the main danger is intraoperative bleeding. In this new technique the ductus is sutured in continuity before division. The use of this technique reduces the risk of operative haemorrhage.

\section{Description of the technique}

The initial steps of exposure and dissection of the ductus are the same as in the standard operation of division and suture. Touroff's (1942) technique of dissecting the ductus is usually followed. When the ductus has been cleared all round, two pairs of Cooley's ductus clamps are applied, one close to the pulmonary artery and the other close to the aorta. Two separate rows of continuous horizontal mattress sutures are inserted between the two clamps as far apart as possible using $16 \mathrm{~mm}$ round-bodied double-armed 4/0 polypropylene sutures, starting with the side of the ductus furthest from the surgeon (Fig. 1). When both rows of horizontal mattress sutures are in place the four needled ends of the sutures are held up to avoid any loose loop in the ductal area. The ductus is then divided between the two horizontal suture lines (Fig. 2), and the same needles are used to suture the cut ends with a continuous over-and-over stitch (Fig. 3). Initial placement of the horizontal mattress sutures prevents the divided ductal ends from slipping. These horizontal mattress sutures also provide a stronger hold for the final over-and-over stitching. There has been no problem of leakage from the stitch holes.

This technique has been used in both small and large ducts in children and adults without difficulty. There may be a criticism that while the ductus is being divided one of the sutures may be

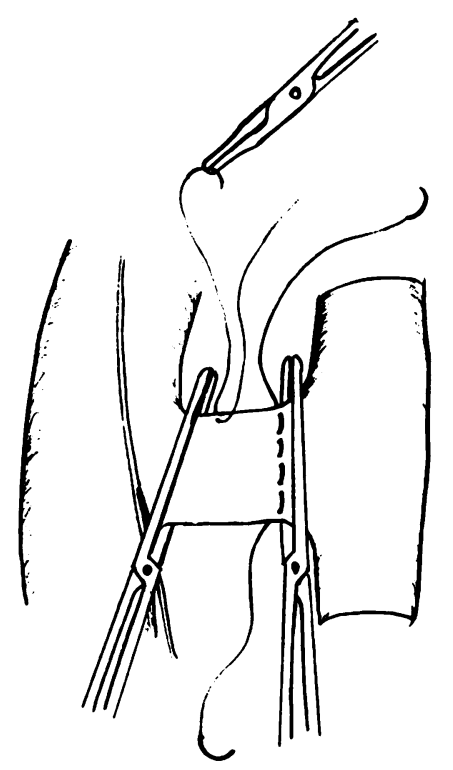

Fig. 1 Ductus dissected: continuous horizontal mattress sutures being applied between two ductus clamps. 


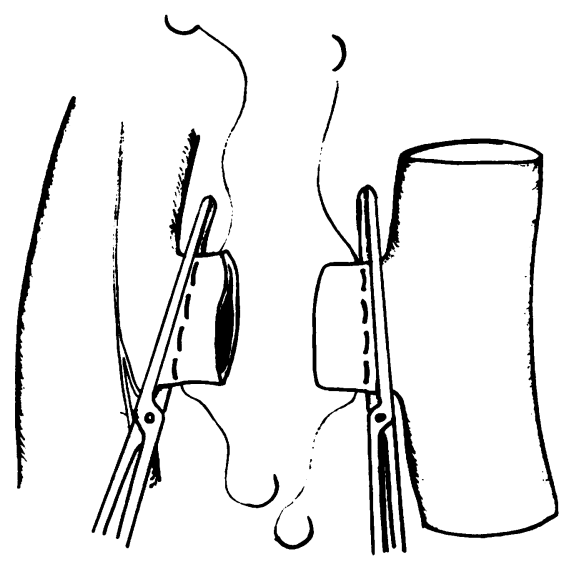

Fig. 2 Ductus divided between two horizontal mattress suture lines. Second row of over-and-over continuous stitching in progress.

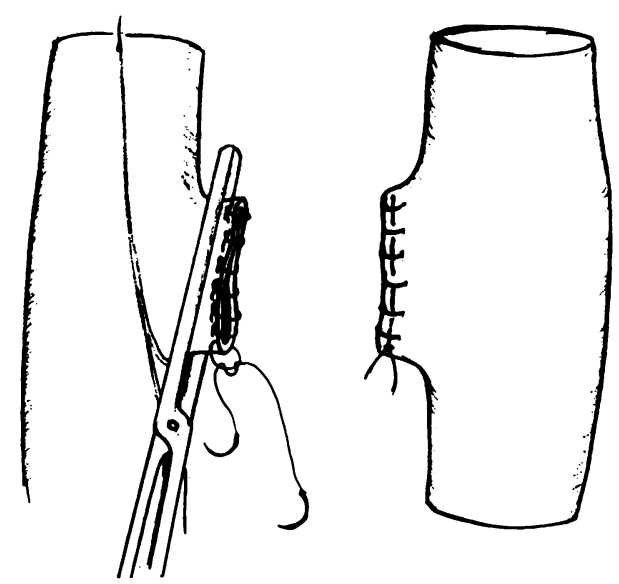

Fig. 3 Divided ends after completion of sutures.

accidentally cut. This has not so far happened. It could in any case be avoided by the use of several interrupted mattress sutures. The suturing can be finished by inserting a second row of continuous over-and-over stitches.

\section{Advantages of the new technique}

By suturing the ductus in continuity before dividing it one can minimise the problem of bleeding and its consequences. If there is bleeding, especially from the pulmonary end of the divided ductus, the recurrent laryngeal nerve, which may be included in stitches that are applied blindly to control the bleeding, is in jeopardy. The initial suture lines prevent the divided ductal ends from retracting under the clamps. They also provide a firm hold for the final over-and-over stitching, which is particularly important if a ductus is fri- $\overrightarrow{i r}$ able from atheromatous change or previous 을 infection.

\section{Results}

This technique of 'suture, division, and suture' has been used in 29 patients since the middle of $\vec{\theta}$ 1973. The ages of the patients ranged from $2 \forall$ months to 33 years. There were 19 children and 10 adults. The size of the ductus varied from $8 \mathrm{~mm}$ to $21 \mathrm{~mm}$ in diameter. The operative blood loss in these patients was as follows: under $30 \mathrm{ml}$ in 11 (10 children and 1 adult), under $50 \mathrm{ml}$ in 8 ( 7 children and 1 adult), under $80 \mathrm{ml}$ in 6 (4 adults and 2 children), and in 4 adults the blood loss ranged from $100 \mathrm{ml}$ to $110 \mathrm{ml}$. There has been no instance of postoperative complication.

I thank Professor G. B. Ong, Head of the Department of Surgery, for advice and encouragement. The figures were drawn by Dr. M. Grant.

\section{References}

Gross, R. E. and Hubbard, J. P. (1939). Surgical ligation of a patent ductus arteriosus. Journal of the American Medical Association, 112, 729-731. Touroff, A. S. W. (1942). A modified technique of surgical ligation of patent ductus arteriosus. Sur- N gery, 12, 24-30.

Requests for reprints to: Dr. P. Nandi, Lecturer in $\underset{\omega}{N}$ Surgery, University of Hong Kong, Queen Mary $\sigma$ Hospital, Hong Kong. 